\title{
Technological innovation for prehospital stroke triage: ripe for disruption
}

\author{
Juan Carlos Martinez-Gutierrez 다, ${ }^{1}$ Ronil V Chandra, ${ }^{2}$ Joshua A Hirsch ํㅏ, ${ }^{3}$ \\ Thabele Leslie-Mazwi ${ }^{1,4}$
}

${ }^{1}$ Neurology, Massachusetts General Hospital, Boston, Massachusetts, USA ${ }^{2}$ Interventional Neuroradiology, Monash Health, Monash University, Clayton, Victoria, Australia

${ }^{3}$ Radiology, Massachusetts General Hospital, Boston, Massachusetts, USA ${ }^{4}$ Neurosurgery, Massachusetts General Hospital, Boston, Massachusetts, USA

Correspondence to Dr Juan Carlos MartinezGutierrez, Department of Neurology, Massachusetts General Hospital, Boston, MA 02114, USA; jmartinezgutierrez@mgh. harvard.edu

Received 3 March 2019 Revised 21 May 2019

Accepted 22 May 2019 Published Online First

14 June 2019

\section{Check for updates}

(c) Author(s) (or their employer(s)) 2019. No commercial re-use. See rights and permissions. Published by BMJ.

To cite: MartinezGutierrez JC, Chandra RV, Hirsch JA, et al.

J Neurolntervent Surg 2019:11:1085-1090.

\section{ABSTRACT \\ Background With the benefit of mechanical} thrombectomy firmly established, the focus has shifted to improved delivery of care. Reducing time from symptom onset to reperfusion is a primary goal. Technology promises tremendous opportunities in the prehospital space to achieve this goal.

Methods This review explores existing, fledgling, and potential future technologies for application in the prehospital space.

Results The opportunity for technology to improve stroke care resides in the detection, evaluation, triage, and transport of patients to an appropriate healthcare facility. Most prehospital technology remains in the early stages of design and implementation.

Conclusion The major challenges to tackle for future improvement in prehospital stroke care are that of public awareness, emergency medical service detection, and triage, and improved systems of stroke care. Thoughtfully applied technology will transform all these areas.

\section{INTRODUCTION}

With proof established about the profound and growing benefit of mechanical thrombectomy (MT) for acute ischemic stroke, the imperative to effectively identify emergent large vessel occlusions (ELVOs) has never been greater. ${ }^{1}$ To maximize treatment potential we need to reduce symptom onset to reperfusion time. This is largely influenced by prehospital and in-hospital processes. ${ }^{2}$ Most current prehospital operational algorithms for suspected stroke require that emergency medical services (EMS) take patients to the nearest hospital. Components of this current system must, in various ways, evolve.

A recent meta-analysis evaluating stroke workflow categorized prehospital care broadly as care prior to a comprehensive stroke center (CSC), where thrombectomy would occur. ${ }^{3}$ This definition therefore encompassed prenotification, mobile stroke units, ship and drip instead of drip and ship, application of vessel imaging at the primary stroke center (PSC), cloud-based imaging sharing between the PSC and CSC, tele-stroke application at the PSC, and air versus ground transfer. Despite this broad definition only 11 studies focused on prehospital processes were available, but meta-analysis detected a highly significant weighted mean difference of $37 \mathrm{~min}$ time saved for implementation of workflow interventions. Time saved translates into disability-free life for our patients. Many of the included studies were retrospective cohort studies and did not leverage the full power of technological innovation.

This current review applies a more focused definition to 'prehospital', specifically the use of technology prior to arrival at any healthcare facility (either a PSC or CSC). By this definition, the current use of prehospital technology to produce improvements in endovascular stroke triage is fledgling and variable in application across the country. There is therefore significant potential to leverage technology in the prehospital arena to refine stroke care for ELVO.

Lessons from history for prehospital technologies Lessons from the history of prehospital technologies and systems of triage can inform current efforts to develop novel improvements. A ready example is ambulances themselves. The first true ambulances developed during the eighteenth-century Napoleonic era, when trained medical staff accompanied wounded soldiers to field hospitals with the goal of stabilization to save lives until better care could be accessed. In 1894 the first motorized electrical ambulance was developed in St. Louis and by the early twentieth century more effective gasoline-powered vehicles manufactured specifically as ambulances began to become widespread. These vehicles belonged to hospitals and were being deployed in a limited manner. It was only in 1967 that the US government established the 911 call system, another revolutionary technological advancement that allowed for swift and more efficient delivery of emergency medical care.

A second example of highly successful prehospital technology is field electrocardiography (EKG), which has driven dramatic process improvement in prehospital care. While approximately half of patients with acute myocardial infarction have ST segment elevations (STEMI), first responders using computer-assisted EKG have up to a $98.7 \%$ specificity and $92.8 \%$ sensitivity for identifying in the field these candidates for reperfusion therapy. ${ }^{4}$ Having early diagnostics allows for earlier activation of interventional teams and subsequently more reperfusions, faster reperfusions, shorter ED triage, and shorter door-to-balloon times. ${ }^{4}$ This clearly identified advantage and the ease of use of EKG led to widespread adoption. Paramedics now all receive training in STEMI identification, and currently many ambulances possess the on-board capacity of transmitting EKGs to the center they 


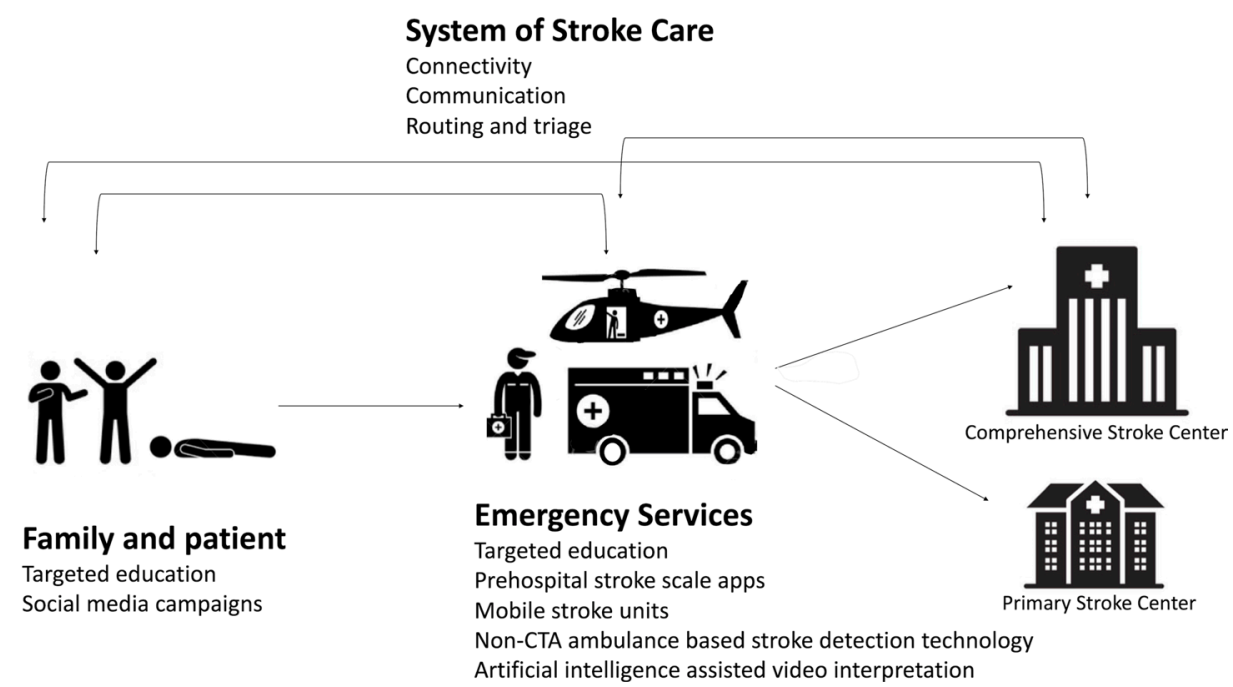

System of Stroke Care

Connectivity

Communication

ting and triage

Artificial intelligence assisted video interpretation

Figure 1 Targets for application of prehospital technology. There is broad utility for technology across the entire spectrum of prehospital stroke care.

are triaging as well. With STEMI guidelines establishing a goal of first medical contact-to-device time of $90 \mathrm{~min}$ or less, states incorporated legislation for EMS to enable field bypass to take patients directly to Percutaneous Coronary Intervention (PCI)-capable centers. ${ }^{5}$ This influx of higher patient volume drove highly specialized centers to become even more proficient, with resulting superior survival and functional outcomes.

These examples translate well to ELVO and the application of prehospital technology, with many translatable lessons. It is also clear that the adoption of such technology will likely be an iterative process, and therefore a sustained effort is required to reach optimal potential of these technologies. Figure 1 summarizes potential targets for prehospital technological advances.

\section{Current prehospital challenges}

Despite limited studies evaluating prehospital ELVO care, in the prehospital space certain key requirements hold at three levels of care:

1. The public: better public awareness about symptoms and treatment options for ELVO to ensure rapid activation of EMS services.

2. EMS: better ability for EMS to detect and grade stroke severity

3. System of stroke care: improved triage of ELVO patients to centers that can provide the care they need. More cohesive coordination of the entire stroke, and specifically ELVO, care system.

Technology has the power to aid our communal efforts in all these components.

Technology is broadly considered the application of scientific knowledge to the practical aims of human life. History provides countless examples of the transformative change technology brings, from the invention of the wheel to personal computing. With expanded technological capability and its widespread integration, we are uniquely positioned to leverage technology. In the following review we discuss some of the current technological options and promise in the prehospital care of ELVO patients, while acknowledging that it remains a field in constant evolution.

\section{Technology and the public}

While faster triage and faster alarm-to-treatment times are important areas of improvement, tremendous gains can be made to reduce symptom-to-alarm times through public education. Studies suggest that up to $40 \%$ of the population cannot name more than one stroke symptom, with even lower rates among the elderly. ${ }^{6}$ Moreover, despite recognition of stroke symptoms, patients or bystanders do not necessarily consider calling 911, as evident in the NINDS t-PA study where only $45 \%$ of patients presented through EMS. ${ }^{7}$ Currently only about a quarter of stroke patients arrive within the tPA window. ${ }^{8}$

Despite promising studies on public education campaigns through television, radio, print media, and actual sit-down sessions, public stroke education has remained low. ${ }^{8-11}$ Nevertheless, most studies have been conducted before the wide adoption of social media. Consider the technology of the two largest social media platforms. With 2.32 billion monthly active users on Facebook and 300 million on Twitter, the capacity to reach billions of future bystanders or patients at a global level through social media is unprecedented. The role of 'influencers', targeting individuals whose popularity directs social media attention to particular causes, is an area yet to be explored for stroke. Prior television-based advertising campaigns demonstrate the effectiveness of public education, and highlight the need for persistence to enact durable change.

Technology harvests public information and enables targeted advertising to those most at risk, by geography, purchasing habits, behaviors, and so on. Technology can further target the education of families or caregivers of high-risk individuals, producing greater efficiency for outreach efforts. For example, the Duke Health System has technology-based initiatives aimed at disseminating their 'BEFAST' initiative to barber shops and church groups, identified as gatherings of higher-risk individuals. ${ }^{12}$

\section{Technology and EMS}

In the era of thrombectomy, the ability of EMS to not just detect but grade stroke severity has gained new importance. Technology enabling this ability through EMS workforce education, simplifying the assessment of stroke, and providing tools for triage decisions represent key solutions.

\section{EMS education and stroke scale Apps}

Technology-based educational solutions for EMS detection of stroke are becoming available. Technology enables the efficient education of broad groups of EMS providers, important given the large number of EMS agencies in each state. The use of 
online CME and video education for EMS education (eg, the FAST-ED video from the American Heart Association) allows EMS provider education at timing convenient for them, and the ability to perform pre- and post-education testing to assess retention. $^{12} 13$ The current absence of mandatory requirements for ELVO education courses represents a future target for improved EMS knowledge.

The large clinical deficits induced by ELVO provide an opportunity for EMS to identify potential ELVO patients on scene. A variety of prehospital ELVO stroke scales exist, most benchmarked against the NIHSS and simplified in formats with some such as the RACE scale validated in the field. ${ }^{14}{ }^{15}$ A variety of apps to aid prehospital ELVO detection exist, including single scale (eg, the RACE Scale app) and more comprehensive (eg, the SNIS Stroke Scales app) apps. Technology has allowed an additional step, with apps that provide both diagnostic assistance and decision or triage support to EMS crews. For instance, the FAST-ED App not only calculates stroke severity with the FAST-ED scale but also uses real-time GPS data to direct EMS to the most accessible endovascular capable centers. ${ }^{16}$ However, the scales lack specificity and have limited data about real-world application, highlighting the work that needs to yet be done before their widespread acceptance.

\section{Mobile stroke unit technology}

The current standard of thrombolysis for stroke is largely limited to tPA administration within the first 4.5 hours. Only about $3.4 \%$ to $5.2 \%$ of stroke patients receive tPA in this window. ${ }^{17}$ The mobile stroke unit (MSU) has emerged as a solution to providing early, on-scene intravenous thrombolytic delivery and, relevant to ELVO, modify triage of stroke. Mobile stroke units, conceived in 2003, were first piloted in 2010 in Germany. ${ }^{18}{ }^{19}$ What distinguishes this ambulance-based system is an on-board CT scanner, point of care laboratory, and telemedicine transmission technology. The point of care laboratory has the capacity to perform stroke-relevant laboratories including CBC, INR, PTT, and glucose. The imaging hardware consists of portable, radiation-shielded CT scanners. The ambulance must be parked and still for image acquisition to occur. This technology allows suspected ELVO patients to bypass primary stroke centers and be transported to centers capable of MT, but still realize the benefit of early tPA administration. Activation of the MSU varies in criteria between locations, with a key component being triage of potential strokes through telephone-based questionnaires for the 911 dispatcher. Units are active in many US cities and across the world. ${ }^{20}$

Certain MSUs have taken imaging a step further, acquiring CTA imaging of the head and distal neck. ${ }^{21}$ This allows confirmation of the presence of ELVO, and mobilization of resources at the CSC to receive and treat the patient immediately on arrival. CTA imaging is not widespread technology yet in MSUs currently, given the additional challenges this carries (contrast storage, injector technology, risk of allergic reactions, and so on).

While not specifically focused on the prehospital space, teleradiology concepts with special focus on stroke hold promise for MSUs. Applications such as i-Stroke (only available in Japan), ResolutionMD, and VizAI provide smartphone platforms for image sharing. With the advent of artificial intelligence, companies such as Brainomix and VizAI have developed novel algorithms for the CT and CTA analysis. Software such as e-ASPECTS has been successfully integrated into the MSU workflow, and it is likely that further integration of automated interpretation will occur in MSUs. ${ }^{22}$
While studies have been able to demonstrate reductions in alarm-to-treatment times of up to $25-35 \mathrm{~min}$ and increased thrombolysis rates by as much as $12 \%$, long-term outcomes of these cohorts have not been significantly different than their controls, particularly for excellent outcomes (mRS 0-1). ${ }^{23-26}$ For mRS $0-3$ and for mortality there may be advantages, suggesting that mRS shift may be a better measure to assess the impact of MSU. ${ }^{26}$ Data for the impact for ELVO patients is limited: a small 10-patient observational study looking at MT metrics with MSUs demonstrated reductions in CT to MT time (82 vs $165 \mathrm{~min}$ ) when compared with hospital-to-hospital transfers. ${ }^{27}$

While the promise of MSUs continues to be assessed, their technological limitations are being actively tackled. The quality of CT imaging (including better image stabilization to permit scanning while driving), high fidelity images, remote clinical evaluation, and transmission of radiological data continue to pose challenges. Clearly demonstrated cost-effectiveness will likely catalyze wider adoption. The unique strength of this technology over most others is the capability for earlier and, potentially, more frequent treatment of stroke.

\section{Other ambulance-based stroke detection technology}

While CT and CTA allow direct visualization of brain and blood vessels, a variety of emerging technologies provide alternate approaches for the evaluation of cerebral blood flow and intracranial pressure dynamics in the prehospital environment. The ideal components of a prehospital ELVO detection technology are similar to EKG: simple, cheap, fast, reproducible and reliable, translatable, broadly applicable, accurate, and compact. Furthermore, a binary outcome (eg, 'stroke/no-stroke' or 'transfer to comprehensive center/take to local center') independent of interpretation by EMS would be ideal. ELVO detection is not the sole technological goal. For example, hemorrhage detection, or confirmation of hemispheric involvement, can add specificity and sensitivity to clinical triage.

A variety of different technology is brought to bear in the field of pre-hospital stroke detection, most in the early investigational phases.

\section{Electromagnetic detection}

Cerebrotech visor: This well studied and promising helmet technology uses volumetric impedance phase shift spectroscopy (VIPS) to predict large strokes in approximately $30 \mathrm{~s}^{28}$ VIPS is based on the fact that different brain pathologies have distinct tissue, electrical, and fluid properties. Electromagnetic waves of different frequencies are emitted and then modified by tissue fluid properties, outputting a unique signature. Taking the baseline of the contralateral hemisphere, an asymmetry of $>10 \%$ has been shown to represent large territory damage by stroke or hemorrhage. In a large cohort, this $30 \mathrm{~s}$ test demonstrated a 93\% sensitivity and $87 \%$ specificity for detecting severe stroke (ELVO or ICH $>60 \mathrm{ccs}$ ) from other pathology. The software uses a machine learning platform, and requires no specialized training from the EMS team beyond the basics of application. ${ }^{28}$ The device is being tested in stages: VUE, VIGOR, and FIELD studies are all pending, adding incremental support to the utility of the equipment in the field. ${ }^{29}$

Sense: This helmet device consists of a nine-antennae array that provides 72 data points per scan and a spectrum analyzer coupled to a computer. This allows continuous monitoring to detect neurological assymetry or deterioration. Proof of concept studies are starting for LVO stroke after feasibility studies in animals and ICH patients. 


\section{Accelerometer-based detection}

Brain pulse: The surge of blood into the brain generates asymmetry between hemispheres in the normal state (because the right ICA is closer than the left to the left ventricle) producing 'oscillation' of the brain. Changes in this pattern of oscillation reflect pathology, and the system utilizes a machine learning algorithm to identify and refine its ability to determine exactly what the pathology is. A helmet is applied to the patient, and the recording and interpretation takes approximately 4 mins to complete.

\section{Microwave detection}

StrokeFinder MD100: This device applies low-energy microwave technology transmitted through the brain. ${ }^{30}$ Microwaves scatter over a minute within the brain (as opposed to X-rays passing through or sound waves bouncing back), and detection of scatter through eight strategically positioned antennae queries the state of the underlying tissue. In addition, it uses a proprietary machine learning-based algorithm based on healthy participant data and geared to differentiating ICH and ischemic strokes. The proof of concept study could differentiate 30\% to 65\% of ischemic strokes from $\mathrm{ICH}$, depending on the sensitivity threshold for ICH.

\section{Infrared detection}

Infrascanner 2000: This handheld near-infrared technology gathers differential light absorption data at specified landmarks in the skull (left and right frontal, parietal, occipital, and temporal). This $2-3 \mathrm{~min}$ test is specifically designed for hematoma detection in traumatic brain injury (TBI) with no data yet available for ischemic stroke. ${ }^{31}$

\section{EEG based detection}

Brainscope One: This device uses a modified frontal chain of five electrodes to input 5-10 mins of data into a structural brain injury algorithm with binary output of positive or negative. In a stroke/ICH and stroke-mimic population, it showed a $91.7 \%$ sensitivity and $50.4 \%$ specificity for identifying strokes. $^{32}$

Alphastroke: Designed as a handheld device the size of a cellphone, this technology similarly uses EEG leads to detect asymmetry between the two hemispheres. Recording occurs over approximately a minute and the outcome is a binary decision of stroke or not.

\section{Ultrasound detection}

Lucid and NeuralBot: This TCD system evaluates MCA flow bilaterally using a robotic gantry. The robotic arms have $5 \mathrm{df}$ to enable finding an MCA signal. The manufacturer envisions potential applications in stroke, TBI, ICH, SAH, dementia, and migraine, though data is still being collected. The device is FDA cleared and CE marked from May 2018. ${ }^{33}$

Notably, in considering these technologies there is no literature available yet for comparative evaluations of these devices. Additionally, manufacturers are often reticent concerning technical details, limiting available information. Limitations of these novel devices are both potentially low specificity in realworld applications and concerns regarding cost-effectiveness. Nevertheless, the potential for one of these to be an 'EKG of the brain' in the future is real, with every ambulance carrying a version of this technology to assist EMS crews. More development and studies are ongoing to determine the utility of these technologies.

\section{Artificial intelligence to automate clinical stroke detection}

There may be a role for technological aid in direct clinical examination of stroke. Using artificial intelligence (AI) to interpret video images has been demonstrated for still images. ${ }^{34} 35$ The goal of educating EMS on clinical identifiers is important, but stroke frequency is such that cases per EMS provider, and therefore experience per EMS provider, is limited. The possibility to automate the process may make it highly accurate. Facial weakness detection, for example, requires a sequential process: identify the face, use landmarks to focus on the mouth and surrounding area, identify asymmetry, train an algorithm to recognize that asymmetry and distinguish normal from abnormal, and then integrate that technology into video. That process would need to be duplicated for limb weakness, and speech and language abnormalities. The goal is that EMS film a patient video using a handheld device and then receive an analysis of what the AI algorithm has detected regarding stroke probability. This technology remains very much in its infancy, requiring extensive training of the algorithm with normal and abnormal patients. Considering the potential for better EMS detection of stroke, this holds promise for future application.

\section{Technology and systems of stroke care}

Nationally, ELVO and stroke care show high variability in system processes. Access to care varies geographically, by hospital factors, and by patient factors, making coordination of care a major challenge. ${ }^{36}$ Delivering high complexity acute care to large population bases requires strong networks of collaboration, with communication between hubs and spokes. ${ }^{37}$ In addition to the network itself, external, variable factors (such as traffic and weather) need to be considered. We know from the cardiac literature that patient transfer is not always to the closest and best hospital. ${ }^{38}$ Given these facts, technology may offer a favorable solution to this major challenge of coordinating systems of care by enhancing communication and facilitating collaboration. As we create or modify networks and systems for prehospital ELVO care, we should consider two critical components:

1. The idealized approach (specifically what are the priorities, rules, and measures for optimization)

2. Using technology to empower us to achieve that idealized approach

\section{Technology enabling connectivity}

Technology drives enhanced connectivity. Despite improvements in EMS equipment, communication between EMS and hospitals continues to rely on outdated radio technology. In order to enhance the coordination of our care systems in stroke, enabling connectivity is essential.

The 911 system is the first link in the chain. Currently, the FCC regulates the strict policies for 911 capabilities for all wireless carriers. By law all service providers must transmit 911 calls to the Public Safety Answering Points (independent of a caller's subscription status. Moreover, the now enhanced E911 rules state that providers are to provide the location of the cell site or base station transmitting such a call, and/or the latitude and longitude of callers when their phone is properly GPS equipped. ${ }^{39}$ Thus, the E911 system holds promise for faster access to possible stroke patients by automating dispatch to specified locations.

Broadband connectivity has revolutionized communication through telestroke. ${ }^{40} 41$ It has been used for remote connectivity in the ambulance setting with adaptation of modems and external antennae as access points to web-based platforms. While 
this advancement is promising, the mobile nature of ambulances challenges this primarily stationary technology. Prehospital telestroke broadband-based platforms have shown prohibitive freezing, poor audiovisual quality, and disconnection rates of up to $20 \%$ during stroke assessments. ${ }^{42}$ Cellular connectivity, meanwhile, has evolved significantly over the past decade, providing a reliable high-quality alternative designed for mobile application. The iTREAT study evaluated the use of a cellular networkbased ambulance telestroke tablet system, demonstrating a 93\% success rate in maintaining continuous 9 min video transmission required for NIHSS assessment. ${ }^{43}$ Another study using an available telemedicine robotic unit on board an ambulance connecting over 4G LTE hotspots demonstrated an $85 \%$ success rate of NIHSS evaluation. ${ }^{44}$ These higher cost systems, while promising, are only recently starting to make inroads with EMS providers.

Management of cost through an episode is increasingly linked to financial performance of many neuroInterventional practices. ${ }^{45}$ For connectivity, lower cost and universally available alternatives include commercially available smartphones that enable routine video connectivity. A Nokia video phone study using $3 \mathrm{G}$ cellular data conducted in 2011 was successful, although the study was performed in a controlled quiet environment with only early-generation, one-way video technology. ${ }^{46}$ Two recent studies have used iPhone 4 devices with the factory standard Facetime application over hospital Wi-Fi networks showing only $6 \%$ poor reception rate and significant interrater reliability of NIHSS scoring. ${ }^{47} 48$ It is easy to imagine that current technology would be even more reliable. Nevertheless, connectivity of economic smartphone platforms over cellular networks in real ambulance settings is yet to be fully determined.

Besides patient video, an important consideration for MSU connectivity is transmission of large image files from CT imaging. To date, industry telemedicine solutions have been employed as methods of transmission of CT digital images in MSUs operating over cellular networks. ${ }^{49}$ Transmission times are not explicitly mentioned in the available literature but door to CT read times are similar in both MSU and ED controls, suggesting rapid connectivity. While some speculate that smartphone video transmission may be of sufficient quality to allow for radiological interpretation, this is yet to be investigated.

Limitations to advancing these technologies will revolve in part around HIPAA compliance. Thus far, studies have relied on servers with encryption, firewall protected hospital Wi-Fi, and basic cellular network data encryption as the primary safety nets. As potential for use of, and demand for, commercially available software and hardware over theoretically unsecured cellular networks is on the near horizon, scrutiny about HIPAA compliance is crucial.

Technology and communication between stroke system components As knowledge and technology improves the sensitivity and specificity of detecting ELVOs, effectively coordinating care maximizes patient access to timely treatment. Technology integration offers a promising solution by facilitating communication and providing analysis of complex data.

The appropriate triage of these cases is pivotal and at times controversial. ${ }^{50}$ Hospital stroke center designation distinguishs centers capable of performing thrombectomy (eg, CSCs) from non-capable centers. However, this may not be widely understood or available to first responders or patients. Moreover, it is not feasible that every suspected stroke patient is taken to the nearest CSC. Despite EMS assessment of clinical severity augmented by the technology previously discussed, there is a complex cost-benefit analysis that needs to occur rapidly when making decisions about EMS routing. Technology promises great assistance with this. ${ }^{51}$

Early communication between EMS and target hospitals has been a revolutionary technological advancement. Radio communication has remained the backbone of emergency medicine communication. Radio prenotification of the hospital by incoming EMS crews allows preparation of hospital staff to deal with complex medical situations such as ELVO, trauma, or STEMIs. Taking advantage of technology advancements, efforts to improve early communication with app-based technologies have started to be incorporated in regional centers. Table 1 summarizes currently available app platforms. Smartphone platform integration allows first responders to pass-off critical

Table 1 Current available apps for integrated prehospital stroke care

\begin{tabular}{llll}
\hline App/developer & Pros & Cons \\
\hline Twiage & Pre-arrival pass-off & Stroke section limited \\
& Transmits images, videos, EKGs & Only prehospital \\
Stop Stroke Pulsara & GPS tracking & Integrates communication between EMS, ED, radiology, neurology, and Interventional team impact on outcomes \\
& Includes stroke scales (LAMS, NIHSS, RACE, FAST/Cincinnati) & Not integrated to EMR \\
& Keeps running track of LSW, tPA times, and MT times & \\
& Alerts when images available & Not universally available \\
& Transmits images & Early phase of deployment \\
& Allows tPA contraindication review by any and all & Lacks data on impact on outcomes \\
CodeStroke Alert & Data (limited) on impact on logistical outcomes & \\
& Clinical score calculators and guidelines & \\
& Instant messaging and read receipts & Interhospital referral \\
& Tracked and timestamped data entries for audit & Not universally available \\
& Real-time GPS tracking & Lacks data on impact on outcomes \\
\hline Join/Allm Inc Japan & Open source & Time stamps events (LSW, CT, MRI, Angio suite, Reperfusion) \\
& Instant messaging platform & \\
\hline
\end{tabular}


information about a patient and send images, videos and EKGs, all while providing real-time GPS tracking for optimized routing and accurate ETAs.

These systems aim to ease critical pass-off and provide information that can guide faster and more accurate activation of response teams, such as neurointerventional teams. Inpatient team communication is facilitated by creating stroke team groups that include EMS, Emergency Department (ED) staff, radiology technologists, neurologists, angiography technologists, and interventionalists. By allowing registration of multiple time points, and alerting to completion of studies or procedures, it further integrates clinically relevant metrics such as LSW, NIHSS, tPA contraindications, and door to reperfusion into the application. Data from the Stop Stroke Pulsara application suggests there is a $20-40$ min decrease in door to needle times. ${ }^{52} 53$ Presumably, door to groin times would be similarly impacted.

While these applications provide a coordinated service it is important to acknowledge the communication currently undertaken by teams across the country using text message-based groups that incorporate EMS, ED, and stroke or neurointerventional teams. This occurs through iMessage, WhatsApp, and a variety of other text-based platforms. These allow users to create groups, exchange real-time text messages, send and receive pictures and video, and therefore function more effectively. HIPAA concerns require constant vigilance about the content being exchanged, but the existence of these messaging-based options indicates the appetite for such technology in the prehospital stroke space. Further testing and studies are needed to inform the impact of apps in outcome measures.

\section{Technology for route assistance}

Health service organizations and governments are increasingly focused on design and delivery of hyperacute stroke services to optimize patient access. Bypass models are being actively tested but it is likely that there is no one-size-fits-all approach to where a patient picked up by EMS is taken (drip-and-ship versus bypass to CSC). ${ }^{545}$ In addition to geography, variables such as mode of transport, traffic conditions, weather, and other considerations come to bear. Technology has already made strides in attacking this major challenge of coordination of care, and holds great promise in further assisting this effort by analyzing live complex data variables to guide prehospital providers.

While siren technology allows for some degree of bypassing traffic, data suggests the average time saving may be only 3 min. ${ }^{56}$ With increased traffic congestion, technology entering the mainstream includes smart traffic lights and intelligent ambulances. Location-based ambulance signaling could change path lighting to green for faster transit, for example.

A proof of concept study used Google AI to evaluate the Google Map platform for modeling such variables as traffic in a major metropolitan area. ${ }^{57}$ Google Map technology became a mobile app platform in 2008 , but incorporated the crowdsourcing Waze app in 2013, unlocking further technological potential. Transport information is therefore available in real time from vast numbers of users, which, combined with smart sensors and information from local authorities, allows optimization of transport timing for a particular route. For ELVO, the vision of such a system would be to inform EMS crews in the field of the most direct routes in real time. ${ }^{57}$ This information would include how to handle a patient (eg, bypass to CSC vs drip-and-ship decisions for patients in watershed geographies), which CSC/thrombectomy-capable center to choose (eg, incorporating local door-to-needle and door-to-groin times as well as traffic conditions), and the best routes to get there. Technological integration to that extent would make the prehospital ELVO stroke network a dynamic and flexible construct and ensure each patient has optimized and bespoke triage.

\section{CONCLUSIONS}

The major challenges in prehospital stroke care are related to informing the public, enabling and empowering EMS, and improving and modifying the system of stroke care. Technology offers great promise in providing solutions to address these challenges. Many potential targets exist, with variable progress at these various levels. Currently, EMS-based technologies have demonstrated the greatest development and implementation. Technology is being applied to the major challenges of stroke detection and grading, as well as coordination of stroke care. Technology-based EMS education and the use of internet-based connectivity have become realities. Further efforts to use novel devices for improving both detection and triage of patients are fledgling, far from becoming standard of care. However, the reality of widespread, compact, ambulance-based stroke detection equipment to augment the clinical examination may be only a few years away.

Enhancing connectivity through app-based platforms has begun to evolve how we provide coordinated care. There are already simpler versions of these solutions being used through direct smartphone texting platforms. Clearly, the appetite for better solutions is large. Coordinated, system approaches to ELVO triage, lubricated and driven by technology, could dramatically impact patient outcomes. If PSC bypass is a technique with proven benefit in ELVO care, as many expect, there will be a powerful incentive for more prehospital technologies to leap forward, because of service demand.

Ultimately, our collective ability to harness and leverage the power of technology is a keystone in our prehospital efforts to improve patient care for ELVO.

Contributors JCMG and TLM wrote the original draft. All authors had an opportunity to check for accuracy, make editorial suggestions, and approved the final draft.

Funding The authors have not declared a specific grant for this research from any funding agency in the public, commercial or not-for-profit sectors.

Competing interests JAH consults for Medtronic, is on a DMC for Relievant and a DSMB for Cerenovus. JAH receives grant support from the Neiman Health Policy Institute.

Patient consent for publication Not required.

Provenance and peer review Not commissioned; externally peer reviewed.

\section{REFERENCES}

1 Leslie-Mazwi T, Chandra RV, Baxter BW, et al. ELVO: an operational definition. J Neurointerv Surg 2018;10:507-9.

2 Fifi JT, Dabus G, Mack WJ, et al. In the thrombectomy era, triage in the field improves care. J Neurointerv Surg 2018;10:607-8.

3 Janssen PM, Venema E, Dippel DWJ. Effect of workflow improvements in endovascular stroke treatment. Stroke 2019;50:665-74.

4 Chartrain AG, Kellner CP, Mocco J. Pre-hospital detection of acute ischemic stroke secondary to emergent large vessel occlusion: lessons learned from electrocardiogram and acute myocardial infarction. J Neurointerv Surg 2018;10:549-53.

5 Jneid $\mathrm{H}$, Addison D, Bhatt DL, et al. AHA/ACC clinical performance and quality measures for adults with ST-elevation and non-ST-elevation myocardial Infarction: a report of the American College of Cardiology/American Heart Association Task Force on Performance Measures. J Am Coll Cardiol 2017;70:2048-90.

6 Fogle CC, Oser CS, Troutman TP, et al. Public education strategies to increase awareness of stroke warning signs and the need to call 911. J Public Health Manag Pract 2008;14:e17-22.

7 Barsan WG, Brott TG, Broderick JP, et al. Urgent therapy for acute stroke, 1989

8 Tong D, Reeves MJ, Hernandez AF, et al. Times from symptom onset to hospital arrival in the Get with the Guidelines--Stroke Program 2002 to 2009: temporal trends and implications. Stroke 2012;43:1912-7. 
9 Hodgson C, Lindsay P, Rubini F. Can mass media influence emergency department visits for stroke? Stroke 2007:38:2115-22.

10 Reeves MJ, Rafferty AP, Aranha AA, et al. Changes in knowledge of stroke risk factors and warning signs among Michigan adults. Cerebrovasc Dis 2008;25:385-91.

11 Miyamatsu N, Kimura K, Okamura T, et al. Effects of public education by television on knowledge of early stroke symptoms among a Japanese population aged 40 to 74 years: a controlled study. Stroke 2012;43:545-9.

12 DiBiasio EL, Jayaraman MV, Oliver L, et al. Emergency medical systems education may improve knowledge of pre-hospital stroke triage protocols. J Neurointerv Surg Published Online First: 07 December 2018. 10.1136/neurintsurg-2018-014108 2018

13 American CME. Identifying Large Vessel Occlusion (LVO) Strokes with FAST-ED. americancme.com FAST-ED video.

14 Smith EE, Kent DM, Bulsara KR, et al. Accuracy of prediction instruments for diagnosing large vessel occlusion in individuals with suspected stroke: a systematic review for the 2018 guidelines for the early management of patients with acute ischemic stroke. Stroke 2018;49:e111-22.

15 Carrera D, Gorchs M, Querol M, et al. Catalan Stroke Code and Reperfusion Consortium (Cat-SCR). Revalidation of the RACE scale after its regional implementation in Catalonia: a triage tool for large vessel occlusion. J Neurointerv Surg 2018:neurintsurg-2018-014519-2016.

16 Nogueira RG, Silva GS, Lima FO, et al. The FAST-ED App: a smartphone platform for the field triage of patients with stroke. Stroke 2017:48:1278-84.

17 Adeoye $\mathrm{O}$, Hornung R, Khatri P, et al. Recombinant tissue-type plasminogen activator use for ischemic stroke in the United States: a doubling of treatment rates over the course of 5 years. Stroke 2011;42:1952-5.

18 Fassbender K, Walter S, Liu Y, et al. 'Mobile stroke unit' for hyperacute stroke treatment. Stroke 2003;34:e44.

19 Walter S, Kostpopoulos P, Haass A, et al. Bringing the hospital to the patient: first treatment of stroke patients at the emergency site. PLoS One 2010;5:13758-9.

20 Fassbender K, Grotta JC, Walter S, et al. Mobile stroke units for prehospital thrombolysis, triage, and beyond: benefits and challenges. Lancet Neurol 2017:16:227-37.

21 John S, Stock S, Masaryk T, et al. Performance of CT angiography on a mobile stroke treatment unit: implications for triage. J Neuroimaging 2016;26:391-4.

22 Grunwald IQ, Ragoschke-Schumm A, Kettner M, et al. First automated stroke imaging evaluation via electronic Alberta Stroke Program early CT score in a mobile stroke unit. Cerebrovasc Dis 2016:42:332-8.

23 Ebinger $\mathrm{M}$, Winter $\mathrm{B}$, Wendt $\mathrm{M}$, et al. Effect of the use of ambulancebased thrombolysis on time to thrombolysis in acute ischemic stroke. JAMA 2014;311:1622-31.

24 Walter S, Kostopoulos P, Haass A, et al. Diagnosis and treatment of patients with stroke in a mobile stroke unit versus in hospital: a randomised controlled trial. Lancet Neurol 2012:11:397-404

25 Ebinger M, Kunz A, Wendt M, et al. Effects of golden hour thrombolysis: a Prehospital Acute Neurological Treatment and Optimization of Medical Care in Stroke (PHANTOM-S) substudy. JAMA Neurol 2015;72:25-30.

26 Kunz A, Ebinger M, Geisler F, et al. Functional outcomes of pre-hospital thrombolysis in a mobile stroke treatment unit compared with conventional care: an observational registry study. Lancet Neurol 2016;15:1035-43.

27 Cerejo R, John S, Buletko AB, et al. A mobile stroke treatment unit for field triage of patients for intraarterial revascularization therapy. J Neuroimaging 2015;25:940-5

28 Kellner CP, Sauvageau E, Snyder KV, et al. The VITAL study and overall pooled analysis with the VIPS non-invasive stroke detection device. J Neurointerv Surg 2018;10:1079-84.

29 U.S. National Library of Medicine. Volumetric Impedance to Guide Stroke Response (VIGOR).

30 Persson M, Fhager A, Trefná HD, et al. Microwave-based stroke diagnosis making global prehospital thrombolytic treatment possible. IEEE Trans Biomed Eng 2014:61:2806-17.

31 Peters J, Van Wageningen B, Hoogerwerf N, et al. Near-infrared spectroscopy: a promising prehospital tool for management of traumatic brain injury. Prehosp Disaster Med 2017:32:414-8.

32 Michelson EA, Hanley D, Chabot R, et al. Identification of acute stroke using quantified brain electrical activity. Acad Emerg Med 2015;22:67-72.

33 O'Brien M, Ranjbaran M, llyas P, et al. Fully automated transcranial doppler ultrasound insonation of the MCA using a five degree of freedom robotically actuated probe system. Eur J Neurol 2018;25:1-65.
34 Eichler N, Hel-Or H, Shimshoni I, et al. 3D motion capture system for assessing patient motion during Fugl-Meyer stroke rehabilitation testing. IET Computer Vision 2018;12:963-75.

35 Bandini A, Green JR, Richburg BD, et al. Automatic detection of orofacial impairment in stroke University Health Network : Toronto Rehabilitation Institute, Toronto, Canada Department of Speech-Language Pathology, University of Toronto, Canada. 2018:1711-5

36 Kamal N, Sheng S, Xian Y, et al. Delays in door-to-needle times and their impact on treatment time and outcomes in get with the guidelines-stroke. Stroke 2017:48:946-54.

37 Smith EE, Schwamm LH. Endovascular clot retrieval therapy: implications for the organization of stroke systems of care in North America. Stroke 2015:46:1462-7.

38 Iwashyna TJ, Kahn JM, Hayward RA, et al. Interhospital transfers among Medicare beneficiaries admitted for acute myocardial infarction at nonrevascularization hospitals. Circ Cardiovasc Qual Outcomes 2010:3:468-75.

39 Federal Communications Commission. 911 Wireless Services. https://www.fcc.gov/ consumers/guides/911-wireless-services

40 Akbik F, Hirsch JA, Chandra RV, et al. Telestroke-the promise and the challenge. Part one: growth and current practice. J Neurointerv Surg 2017;9:357-60.

41 Akbik F, Hirsch JA, Chandra RV, et al. Telestroke-the promise and the challenge. Part two: -expansion and horizons. J Neurointerv Surg 2017;9:361-5.

42 Van Hooff RJ, Cambron M, Van Dyck R, et al. Prehospital unassisted assessment of stroke severity using telemedicine: a feasibility study. Stroke 2013;44:2907-9.

43 Lippman JM, Smith SNC, McMurry TL, et al. Mobile telestroke during ambulance transport is feasible in a rural EMS setting: the iTREAT Study. Telemed e-Health 2016:22:507-13

44 Wu TC, Nguyen C, Ankrom C, et al. Prehospital utility of rapid stroke evaluation using in-ambulance telemedicine: a pilot feasibility study. Stroke 2014:45:2342-7.

45 Spilberg G, Nicola GN, Rosenkrantz AB, et al. Understanding the impact of 'cost' under MACRA: a neurointerventional imperative!. J Neurointerv Surg 2018;10:1005-11.

46 Gonzalez MA, Hanna N, Rodrigo ME, et al. Reliability of prehospital real-time cellular video phone in assessing the simplified National Institutes Of Health Stroke Scale in patients with acute stroke: a novel telemedicine technology. Stroke 2011;42:1522-7.

47 Anderson ER, Smith B, Ido M, et al. Remote assessment of stroke using the iPhone 4. I Stroke Cerebrovasc Dis 2013;22:340-4.

48 Demaerschalk BM, Vegunta S, Vargas BB, et al. Reliability of real-time video smartphone for assessing National Institutes of Health Stroke Scale scores in acute stroke patients. Stroke 2012:43:3271-7.

49 Kostopoulos P, Walter S, Haass A, et al. Mobile stroke unit for diagnosis-based triage of persons with suspected stroke. Neurology 2012;78:1849-52.

50 Mack WJ, Mocco J, Hirsch JA, et al. Thrombectomy stroke centers: the current threat to regionalizing stroke care. J Neurointerv Surg 2018;10:99-101.

51 Rumsfeld JS, Brooks SC, Aufderheide TP, et al. Use of mobile devices, social media, and crowdsourcing as digital strategies to improve emergency cardiovascular care: a scientific statement from the American Heart Association. Circulation 2016;134:e87-108.

52 Dickson R, Nedelcut A, Nedelcut MM. Stop stroke: a brief report on door-to-needle times and performance after implementing an acute care coordination medical application and implications to emergency medical services. Prehosp Disaster Med 2017:32:343-7.

53 Dickson RL, Sumathipala D, Reeves J. Stop stroke@ acute care coordination medical application: a brief report on postimplementation performance at a primary stroke center. I Stroke Cerebrovasc Dis 2016:25:1275-9.

54 Holodinsky JK, Williamson TS, Demchuk AM, et al. Modeling stroke patient transport for all patients with suspected large-vessel occlusion. JAMA Neurol 2018:75:1477-86

55 Chartrain AG, Shoirah $H$, Jauch EC, et al. A review of acute ischemic stroke triage protocol evidence: a context for discussion. J Neurointerv Surg 2018:10:1047-52.

56 Ho J, Casey B. Time saved with use of emergency warning lights and sirens during response to requests for emergency medical aid in an urban environment. Ann Emerg Med 1998:32:585-8

57 Phan TG, Beare R, Chen J, et al. Googling service boundaries for endovascular clot retrieval hub hospitals in a metropolitan setting: proof-of-concept study. Stroke 2017:48:1353-61.

ORCID iDs

Juan Carlos Martinez-Gutierrez http://orcid.org/0000-0002-6722-8267

Joshua A Hirsch http://orcid.org/0000-0002-9594-8798 